\title{
The feeling of agency: empirical indicators for a pre-reflective level of action awareness
}

\author{
Nicole David ${ }^{1 *}$, Anna Stenzel ${ }^{1,2}$, Till R. Schneider ${ }^{1}$ and Andreas K. Engel ${ }^{1}$ \\ Department of Neurophysiology and Pathophysiology, University Medical Center Hamburg-Eppendorf, Hamburg, Germany \\ Department of Psychology, Westfälische Wilhelms-University, Münster, Germany
}

Edited by:

Christof Koch, California Institute of Technology, USA

Reviewed by:

Ryota Kanai, University College

London, UK

Melanie Wilke, California Institute of

Technology, USA

Uri Maoz, California Institute of

Technology, USA

Nicole David, Department of

Neurophysiology and Pathophysiology,

University Medical Center Hamburg-

Eppendorf, Martinistraße 52, 20246

Hamburg, Germany.

e-mail: ndavid@uke.de

\section{*Correspondence:}

The sense of agency has been defined as the sense that I am the author of my own actions. This sense, however, is usually not reflected upon but instead pre-reflectively experienced. Experimental approaches usually measure the sense of agency by judgments or verbal reports, despite evidence that the sense of agency is not sufficiently assessed on such a reflective level. Here we sought to identify non-verbal measures of the sense of agency, particularly testing the relevance of physiological activity such as skin conductance and heart rate. Manipulating the visual feedback to an executed movement, we investigated how well physiological activity and other movement parameters differed between real and false feedback (i.e., between actual agency and non-agency), and how they related to accuracy of agency judgments. Skin conductance and heart rate did not differ between agency and non-agency situations; neither did they inform agency judgments. In contrast, movement onsets - particularly, discrepancies between feedback and movement onsets - were related to agency judgments. Overall, our results indicate weak visceral-somatic associations with the sense of agency. Thus, physiological activity did not prove to be an empirical indicator for the feeling of agency.

Keywords: sense of agency, action awareness, action monitoring, pre-reflective agency, skin conductance response, heart rate

\section{INTRODUCTION}

The sense of agency refers to the ability to recognize oneself as the author of one's own actions and distinguish these from actions caused or controlled by other sources (Gallagher, 2000). As such, the sense of agency is a key component to self-consciousness (Georgieff and Jeannerod, 1998; Metzinger, 2003; Synofzik et al., 2008a). But what specifically is meant by "sense"? For example, although I am typing these letters on the keyboard, I am more or less aware of myself as the agent of this writing and thinking. Empirical work usually operationalized the sense of agency by self-other action recognition paradigms (e.g., Franck et al., 2001; Farrer and Frith, 2002; Farrer et al., 2003; MacDonald and Paus, 2003; Shergill et al., 2005; Tsakiris et al., 2005; Balslev et al., 2007; David et al., 2007), which imply reflectance upon the actions performed or observed. Our daily actions, however, usually happen in an automated way; we do not necessarily reflect upon them. Although some authors conceptually distinguished between reflective and pre-reflective forms of self-consciousness (Gallagher, 2000; Legrand, 2007), only recently the concept of the sense of agency underwent a similar specification. For example, Synofzik et al. (2008a,b) distinguished between a low-level feeling of agency and a higher-level judgment of agency: the feeling of agency hinges on pre-reflective, sensorimotor processes, whereas the judgment about agency involves reflective or attribution-like processes. More specifically, Synofzik et al. $(2008 \mathrm{a}, \mathrm{b})$ proposed a two-step model, according to which the feeling of agency arises first, only classifying - albeit not at a reflective or meta-representational level - whether oneself was an agent or not based on signals from action execution itself (e.g., efference copies). Second, the judgment of agency classifies who the agent was (similar to Georgieff and Jeannerod, 1998 who system).
Direct empirical comparisons between these two components of agency are rare (e.g., Fourneret and Jeannerod, 1998). At the same time, it is unclear which specific processes inform the feeling and judgment level and how to best assess these levels. Often empirical investigations of agency addressed the latter, manipulating the predicted sensory consequences of performed movements and then asking participants to reflect upon or answer self-other questions of the sort "Was it your movement you saw on the screen?" (e.g., Daprati et al., 1997; Franck et al., 2001; Farrer and Frith, 2002; Farrer et al., 2003; Leube et al., 2003; MacDonald and Paus, 2003; David et al., 2007). Yet, only little is known about empirical indicators for a pre-reflective feeling of agency, independent of any verbal report. As this feeling, according to Synofzik et al. (2008a,b), is heavily based on signals arising from action execution itself, closer inspection of all aspects of motor execution, and control appear a promising avenue (Fourneret and Jeannerod, 1998; Fourneret et al., 2001; Knoblich and Kircher, 2004). What happens, for example, when predicted sensory consequences (e.g., via an efference copy) do not match the observed action outcome? In a seminal experiment, Nielsen (1963) asked his study participants to draw a straight line on a piece of paper while observing their movements via a mirror system. The experimenter, hidden behind a screen, manipulated this visual feedback. He presented his own hand drawing a line, which spatially deviated from the participants' path. As a consequence, participants adjusted their line drawing to the false visual feedback, neither being aware of the other hand nor the adjustments they had made. Later developments of this experiment, which quantified the degree of motor adjustment made to false visual feedback (e.g., by calculating the root mean squared error) and relating it to verbal report, also showed poor conscious access to motor performance 
(Fourneret and Jeannerod, 1998) or a temporal dissociation of motor responses and subjective awareness (i.e., motor adjustments occurred before false feedback was detected; Castiello et al., 1991). Similar dissociations between motor control and awareness in patients (Slachevsky et al., 2001; Bulot et al., 2007; Synofzik et al., 2008) also offer strong support to the two-step feeling-judgment model of agency.

We sought to explore the feeling of agency, testing for further pre-reflective indices, and how these relate to judgments of agency by verbal report. Particularly, we were interested in evaluating the relevance of bodily or peripheral physiological measures for the feeling of agency. Esslen et al. (2008) reported that pre-reflective self-referential processing was associated with electroencephalography activity in areas, which receive homeostatic afferents from somatic and visceral sensations of the body such as the insula and somatosensory cortex - areas also implicated in the sense of agency (e.g., Farrer et al., 2003). Thus, it seemed interesting to investigate a class of physiological indices, which do not directly reflect activity of the central nervous system but visceral or autonomic nervous system (ANS) - a system that functions below the level of consciousness. The ANS controls bodily functions such as heart rate (HR), respiration, perspiration or pupil diameter. Despite being functions of the ANS, they may also be centrally modulated, for example, by stressful or emotional external events (e.g., Malmstrom et al., 1965) and have also been employed in psychological research. Amongst which, (HR, i.e., the number of heartbeats per unit of time) and skin conductance response (SCR; i.e., the electrical conductance of the skin resulting from its moisture or perspiration level) can be considered the most commonly used indices. SCR refers to the phasic increase in conductance in response to a stimulus (Lykken and Venables, 1971). Both, SCR and HR, can be modulated by different states of arousal, emotion, and awareness (Bauer, 1984; Hamm and Vaitl, 1996; Codispoti et al., 2001; Ruiz-Padial et al., 2005; Weike et al., 2007); yet, their role for the sense of agency has not been tested.

Here participants performed simple joystick movements (similar to previous agency experiments: Franck et al., 2001; Farrer and Frith, 2002; David et al., 2007, 2008, 2009) while receiving visual feedback. The visual feedback to their movements was sometimes temporally and spatially manipulated; participants believed to see the experimenter's movements. Participants then rated whether they or the experimenter controlled the visual feedback. The objective of the present study was threefold. We sought to identify indices of the sense of agency in addition to verbal report, while particularly focusing on the relevance of physiological activity. More specifically, we investigated if our dependent variables, such as SCR and HR, differed between real and false feedback conditions (i.e., between factual agency and non-agency) and how they related to the reflective experience of agency. Third, as previous evidence (Shoemaker, 1968; Fourneret and Jeannerod, 1998; Slachevsky et al., 2001; David et al., 2007) suggested that judgments of agency could be subject to errors by misidentification, we aimed to test which other variables were immune to such errors.

\section{MATERIALS AND METHODS PARTICIPANTS}

Eleven right-handed healthy adults (six females; age 19-34 years, mean age 25.7 years) with normal or corrected-to-normal vision participated in the present experiment, in agreement with the
Declaration of Helsinki. Two participants were excluded from further analyses of SCR data and another two from HR analyses because of continuous noise in the raw signal (thus, physiological results refer to $N=9$ ). Before the experiment, each participant signed informed consent. Participants were paid an allowance of $10 € /$ h.

\section{TASK}

Participants were seated in front of a computer screen in an acoustically shielded chamber to perform a simple right-handed joystick (Logitech Attack ${ }^{\mathrm{TM}}$ joystick) task. A cursor appearing at the lower center of the screen signaled participants to initiate their movements toward a target object located at the top of the screen (a white asterisk on a black background; Figure 1A). The target (pseudo-) randomly appeared either on the upper left or right side of the screen. When the target was reached, it was highlighted to indicate target and trial completion. Participants received visual feedback to their movements (real feedback condition) but were told that sometimes they would see the experimenter's movement, who performed the same task outside the chamber (false feedback condition). In reality, false feedback represented previous movement trajectories of the participant, randomly selected from a pool of recorded trajectories and replayed to the participant (David et al., $2007,2008,2009)$. A trial ended when the participants or the false feedback reached the target (on average at $1.3 \mathrm{~s}$ on real or $1.6 \mathrm{~s}$ on false conditions), after which participants judged via button press whether the movement they saw on the screen was self-controlled (1), rather self-controlled (2), rather other-controlled (3), or othercontrolled (4). Participants were naïve about the true nature of the experiment and believed (assessed by informal post-experimental debriefings) that the experimenter performed the same task outside

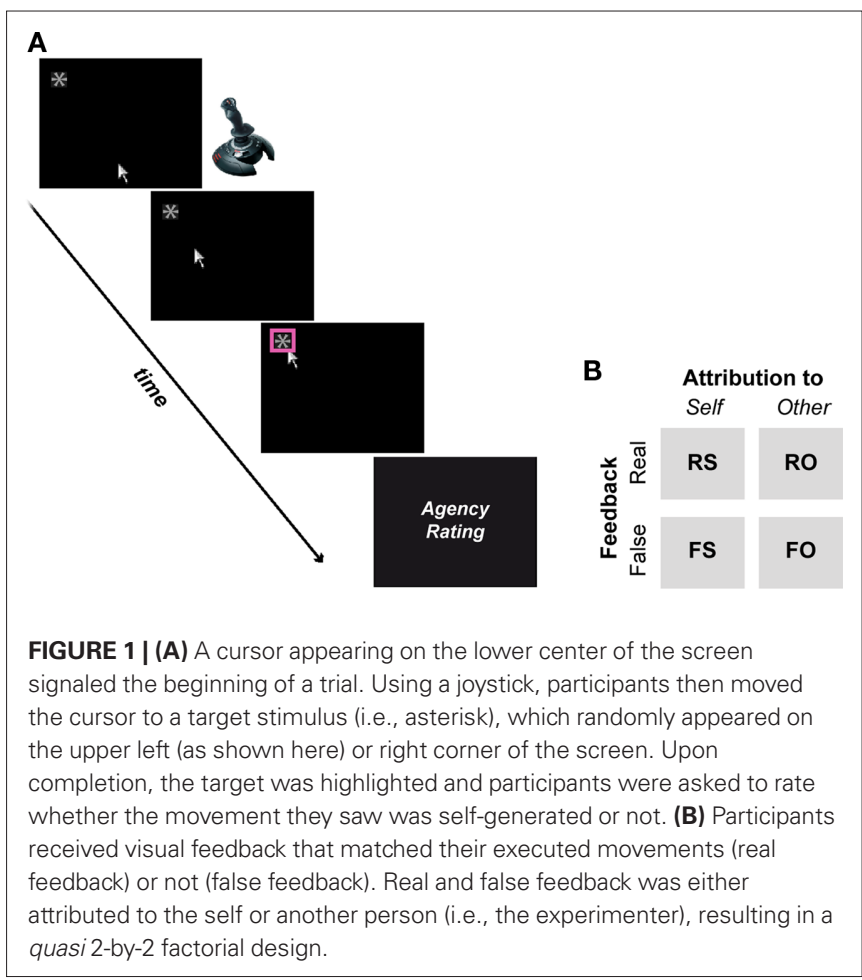


the chamber. Prior to recordings, participants underwent a standardized introduction and practice trial session in order to get used to the trial procedure.

Agency was experimentally manipulated as follows: in 50\% of trials participants saw the corresponding cursor movement to their executed joystick, while the remaining $50 \%$ of trials contained false feedback (160 trials in total). The direction of movements (i.e., left or right-sided target) was pseudo-randomized. The false feedback could deviate temporally (with an inherent minimum delay of $200 \mathrm{~ms}$ ) or spatially from the participant's actual movements (Figure 2). Participants were instructed to react (i.e., initiate their movements) fast and to avoid jerky or diffuse movements. If participants did not move the joystick by $2 \mathrm{~s}$, the trial was aborted. The instruction and $2 \mathrm{~s}$ rule were employed to prevent participants from extremely and voluntarily delaying or distorting their own movements in order to detect false feedback. Note that participants guided their movements blindly during false feedback but reached the target on average in $75 \%$ of false feedback trials. Every 20th trial participants were given the possibility for a self-paced break. The intertrial-interval (i.e., from the end of the rating trials to the beginning of the next joystick movement), allowing peripheral physiological measures to return to baseline, was jittered, and randomized $(10,12,16,18 \mathrm{~s})$. The present paradigm has previously been used in a similar form and has also been described in more detail by David et al. $(2007,2008,2009)$ and Schimansky et al. (2010).

\section{APPARATUS AND RESPONSE MEASUREMENTS}

The task was programmed and administered using the Presentation software (Neurobehavioral Systems, Albany, CA, USA) on a Vision Master Pro 514 monitor (Iiyama, model no. HM204DTA). Monitor refresh rate was $100 \mathrm{~Hz}$ with $1024 \times 768$ screen resolution. The monitor was located $120 \mathrm{~cm}$ in front of participants. To code for
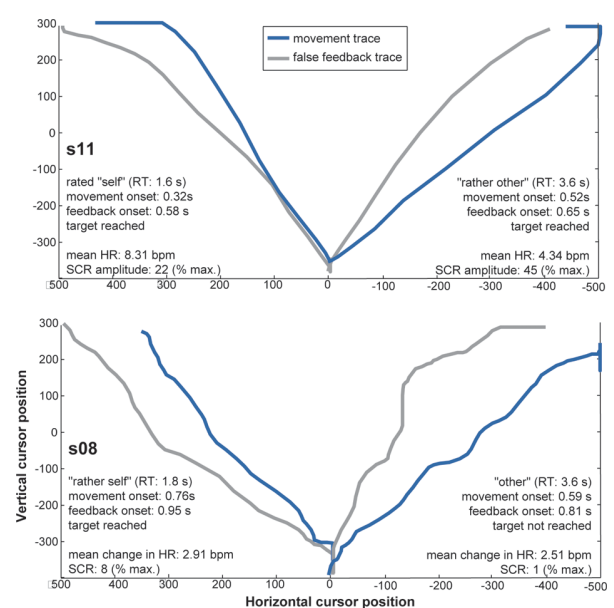

FIGURE 2 | Examples of movement traces plotted against false feedback traces from four different trials and two participants. Corresponding behavioral and physiological measures are entailed in the figures. $x$ - and $y$-axes represent horizontal and vertical cursor positions on the screen. Targets appeared on the left or right upper side of the screen, thus traces to both sides are plotted. This figure does not illustrate dynamic differences in speed between movement and false feedback, which may have informed agency experience. conditions, trial and rating-related parameters, the Presentation software sent event-related trigger to a Biopac system (MP100 unit, Biopac Systems Inc., CA, USA), which continuously recorded the skin conductance and HR.

The SCR was measured in accordance with the procedures described in (Fowles et al., 1981). The SCR signal was recorded from two $\mathrm{Ag}-\mathrm{AgCl}$ electrodes on the index and middle finger of the left hand. A high impedance electrolyte gel with physiological sweat concentrations of sodium chloride $(\sim 0.05 \mathrm{Molar} \mathrm{NaCl})$ was used. A constant electric current was applied and the resultant current flow (in $\mu \mathrm{S}$ ) was measured using the MP100 Biopac unit and the amplifier module GSR100C. The SCR signal was bandpass filtered $(0.05-1 \mathrm{~Hz})$ and sampled at $200 \mathrm{~Hz}$.

The electrocardiogram (ECG) was recorded with the Biopac MP100 system and the amplifier module ECG100C after placing three electrodes on the left and right inner forearm as well as the right lower leg (reference). The ECG signal was bandpass filtered $(0.5-35 \mathrm{~Hz})$ and sampled at $200 \mathrm{~Hz}$. HR was determined from the consecutive $\mathrm{R}-\mathrm{R}$ intervals of the ECG. The Biopac software calculated the interbeat intervals of the ECG using R-top detection, resulting in a HR series consisting of beats per minute (bpm).

\section{DATA ANALYSES BEHAVIORAL DATA}

Dependent behavioral variables comprised (1) agency ratings, which primarily addressed the judgment level of agency. To further characterize the sense of agency beyond verbal reports, also tapping into the sensorimotor feeling of agency, we assessed the (2) time participants initiated their movements (i.e., onset RTs), and (3) the difference in onset of movement and false feedback. Onset RTs on their own have rarely been investigated in agency research despite the possibility that participants may, for example, delay their own movement to test for false feedback. With respect to onset differences between false feedback and movement, other authors have previously investigated who temporally delayed visual feedback is attributed to in terms of agency (e.g., MacDonald and Paus, 2003 used fixed delays between 60 and 270 ms, Franck et al., 2001 delays between 50 and 500 ms, and Farrer et al., 2008 between 50 and $1100 \mathrm{~ms}$ ). Dependent behavioral variables are described in Table 1.

\section{PHYSIOLOGICAL DATA}

The SCR signal was lowpass-filtered at $5 \mathrm{~Hz}$ and baseline-corrected (to $500 \mathrm{~ms}$ before trial onset). Data were cleaned of artifacts (i.e., extreme deflections) by calculating their mean and standard deviation (SD) and excluding all minima or maxima that deviated three SD from the mean. Such artifacts or outlier are rather likely to be due to technical (e.g., cable movement) or other biological reasons (e.g., deep breathing), but do not reflect SCR activity. To distinguish stimulus-related SCR from spontaneous activity, the SCR is usually analyzed in a response window of 1-5 s (Malmivuo and Plonsey, 1995). Thus, any SCR deflection with peak latencies $\leq 1 \mathrm{~s}$ was discarded for further analyses. After preprocessing, on average $88.4 \%$ of trials remained and were used for further statistical analyses of SCR. As large inter-individual variability represents a common limitation of electrodermal measures (Claus and Schondorf, 1999), data were range-corrected by dividing each individual SCR measurement by the participant's maximum response (Lykken and 
Venables, 1971). These normalized individual SCR are plotted in Figure 3. In line with previous research (Bauer, 1984; Hamm and Vaitl, 1996; Weike et al., 2007), peak amplitudes (i.e., magnitudes) per condition were computed as dependent variable for group comparisons (Table $\mathbf{1}$ ).
The HR was baseline- and artifact-corrected similar to the SCR. As the interbeat interval detection performed by the Biopac system was not perfect, trials with bigger jumps in bpm were excluded (if a score was $20 \%$ bigger/smaller than the preceding score; Kettunen and Keltikangas-Jarvinen, 2001). After preprocessing, on average

Table 1 | Dependent variables for the four feedback-by-rating conditions.

\begin{tabular}{lllll}
\hline & RS & RO & FS & FO \\
\hline Number of trials (\%) & $M=45.9, \mathrm{SD}=5.7$ & $M=4.4, \mathrm{SD}=5.7$ & $M=22.7, \mathrm{SD}=6.9$ & $M=27.0, \mathrm{SD}=6.7$ \\
Onset RT (s) & $M=0.60, \mathrm{SD}=0.08$ & $M=0.49, \mathrm{SD}=0.08$ & $M=0.54, \mathrm{SD}=0.08$ & $M=0.54, \mathrm{SD}=0.09$ \\
Onset diff. feedback/movement (S) & $\mathrm{n} / \mathrm{a}$ & $\mathrm{n} / \mathrm{a}$ & $M=0.24, \mathrm{SD}=0.06$ & $M=0.35, \mathrm{SD}=0.08$ \\
$\mathrm{SCR}$ (\% maximum) & $M=13.2, \mathrm{SD}=6.0$ & $M=12.6, \mathrm{SD}=6.4$ & $M=12.9, \mathrm{SD}=6.6 \quad \mathrm{M}=13.5, \mathrm{SD}=7.5$ \\
HR change (bpm) & $M=0.18, \mathrm{SD}=0.74$ & $M=0.56, \mathrm{SD}=1.56$ & $M=0.14, \mathrm{SD}=1.54 \quad M=-0.47, \mathrm{SD}=0.63$
\end{tabular}

$M$, mean; SD, standard deviations; RS, real feedback attributed to self; RO, real feedback attributed to other (i.e., the experimenter); FS, false feedback attributed to self; FO, false feedback attributed to other; RT, reaction time (in seconds); n/a, not applicable; SCR, skin conductance response (percent max. amplitude); HR, heart rate.

* Only data from $N=6$
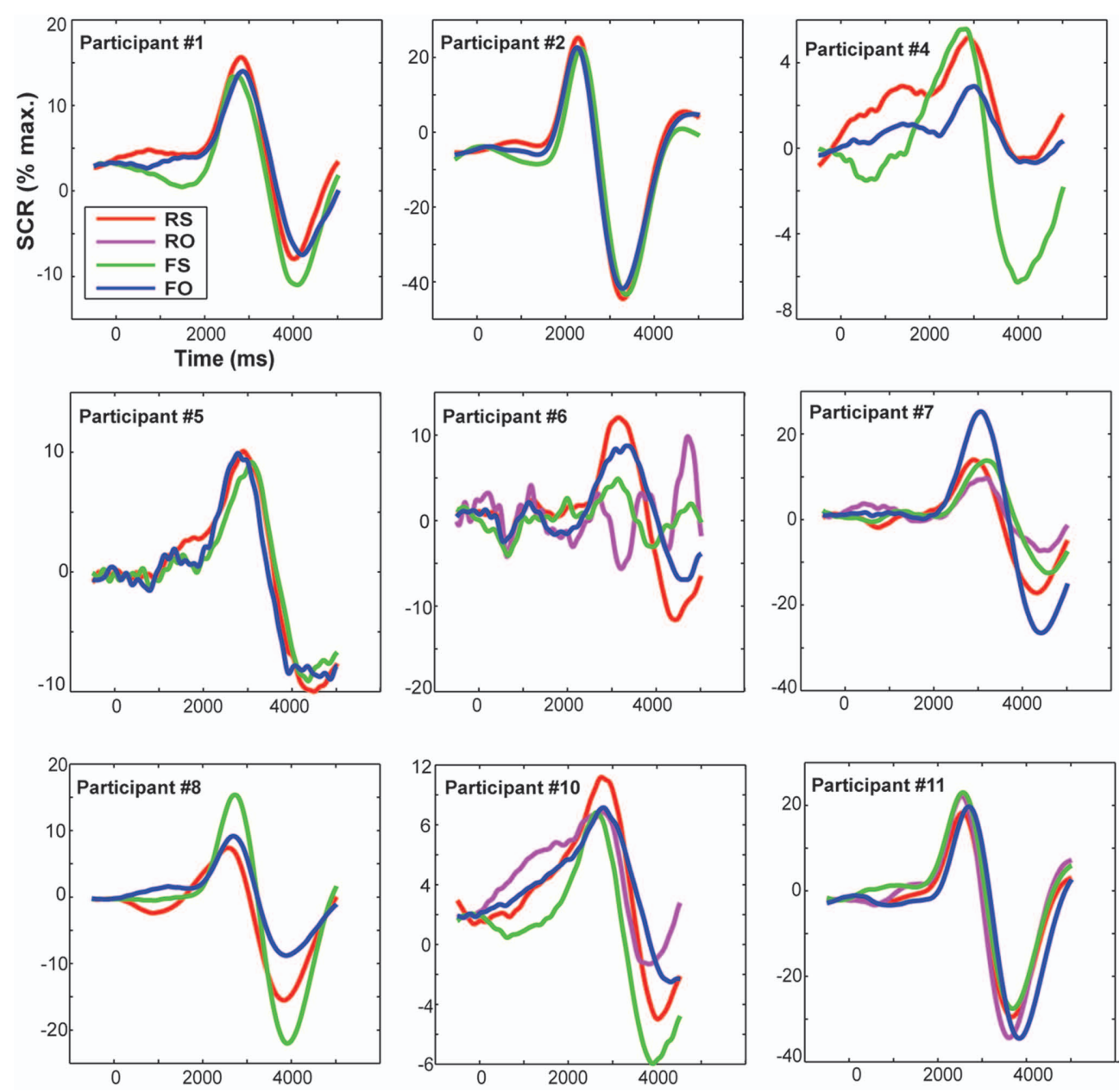

FIGURE 3 | Displayed are individual range- (i.e., normalized to the participant's maximum response) and baseline-corrected SCRs (pre-stimulus baseline was at $\mathbf{- 5 0 0} \mathbf{~ m s}$ ) for each condition. Trial onset was at time zero. RS, real feedback rated self; RO, real feedback rated other, FS, false feedback rated self; FO, false feedback rated other. 
96.7\% of trials remained for subsequent statistical analyses of HR. In line with previous HR research, mean changes in HR over time (at 0.5 to $4 \mathrm{~s}$ post-onset, every $500 \mathrm{~ms}$ ) were computed for each condition and plotted (Codispoti et al., 2001; Ritz et al., 2002; Plesa Skwerer et al., 2009). For statistical analyses an average HR score over time was calculated as dependent variable (Table 1). Because inter-individual variability in physiological activity is usually high, we also show individual HR data in Figure 4.

\section{STATISTICAL ANALYSES OF BEHAVIORAL AND PHYSIOLOGICAL DATA}

To test the effects of our experimental manipulation on dependent variables, a one way MANOVA with the factor Feedback (2 levels: real, false) was performed on the dependent variables: accuracy, onset RTs, SCR and change in HR. In addition, dependent variables were analyzed according to participants' experience of real and false feedback (as self, rather self, rather other, or other). As there were unbalanced and empty cells for some participants (only three rated real feedback as other, only six rather other) and to reduce the amount of multiple comparisons, we collapsed certain and uncertain (e.g., rather self and self) rating bins (see Figure 1B). Onset RTs, onset discrepancy between movement and false feedback, SCR and changes in HR were then compared across the four conditions real feedback rated self (RS), real feedback rated other (RO), false feedback rated self (FS), false feedback rated other (FO) using paired $t$-tests. The Bonferroni-Holm procedure with a global alpha level of
$5 \%$ was used to correct for multiple comparisons. Third, to test for significant relationships between the single measures and accuracy of agency judgments (e.g., accuracy and HR), Spearman's rank correlation coefficient was assessed. All data were analyzed using Matlab (The MathWorks, MA, USA) and the statistical software package SPSS (SPSS Inc., IL, USA).

\section{RESULTS}

\section{AGENCY RATINGS}

There was a significant main effect of feedback manipulation on accuracy of ratings: real feedback was significantly more correctly experienced $(M=91.3 \%$ correct trials, $\mathrm{SD}=11.6 \%)$ as opposed to false feedback $(M=54.1 \%$ correct, $\mathrm{SD}=13.8 ; F(1,20)=46.84$, $p<0.001)$. Table 1 lists the distribution of trials across the four feedback-by-rating possibilities.

\section{MOVEMENT ONSET}

Participants initiated their joystick movements on average at $592 \mathrm{~ms}(\mathrm{SD}=101 \mathrm{~ms})$ on real feedback and $545 \mathrm{~ms}(\mathrm{SD}=86 \mathrm{~ms})$ on false feedback trials. There was no main effect of feedback on onset RTs (i.e., onset RTs for real vs. false feedback; $F(1,20)=1.36$, $p=0.257$, n.s.). Taking into account how feedback was experienced, participants moved significantly later on real feedback trials that were subsequently attributed to themselves, especially as opposed to false feedback that was correctly rated other [RS $>$ FO,
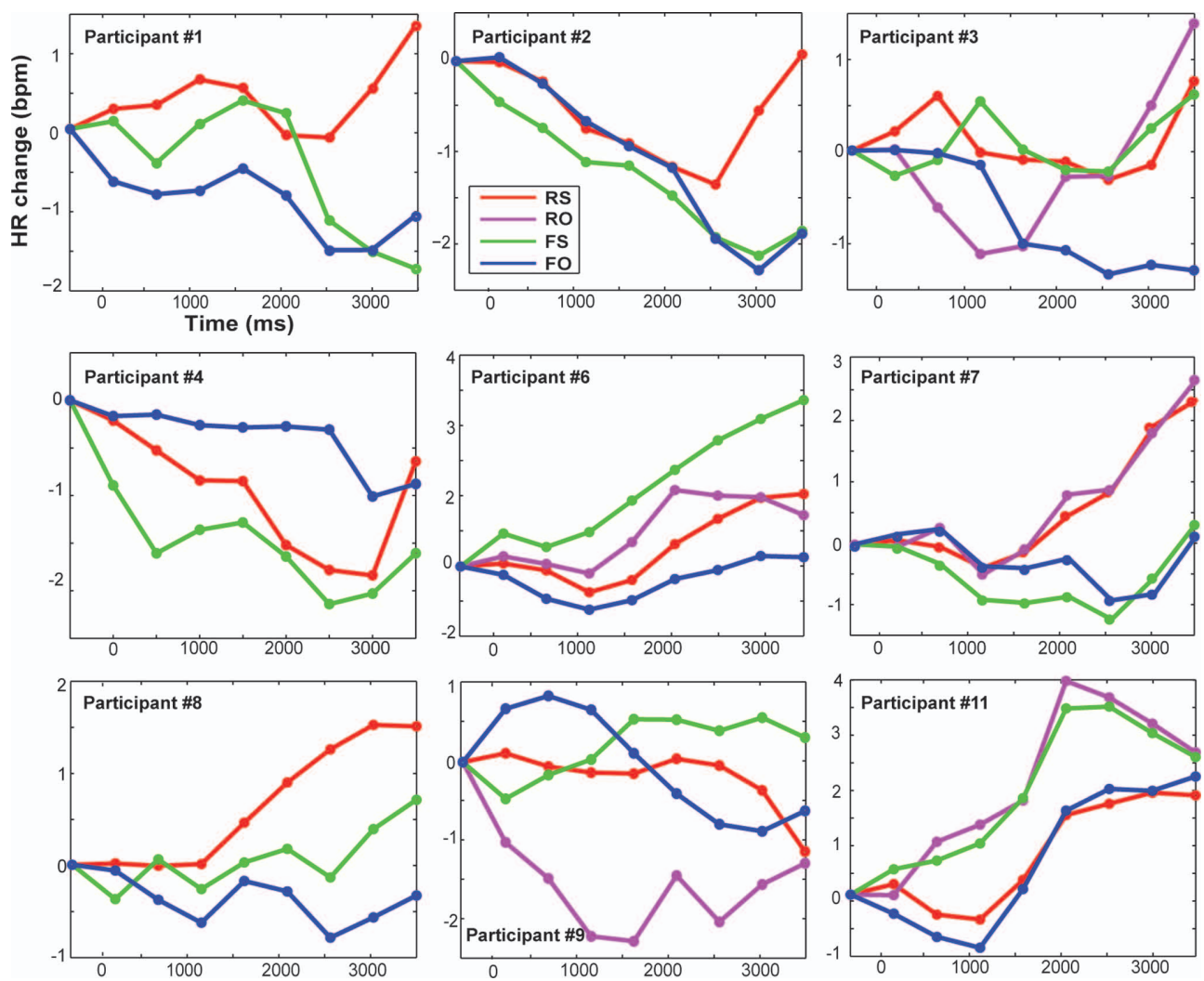

FIGURE 4 | Displayed are changes in heart rate compared to baseline (pre-stimulus baseline was at -500 ms) in beats per minute for each participant and condition over time (averaged over $\mathbf{5 0 0} \mathbf{~ m s}$ time bins). Trial onset was at time zero. RS, real feedback rated self; RO, real feedback rated other, FS, false feedback rated self; FO, false feedback rated other. 
$t(10)=5.04, p=0.001 ; \mathrm{RS}>\mathrm{FS}, t(10)=3.01, p=0.012$, n.s. after correcting for multiple comparisons; Table 1]. There were no other significant differences between conditions with respect to onset RTs. Movement onset did not correlate significantly with overall $\operatorname{accuracy}\left(r_{s}=0.362, p=0.098\right.$, n.s. $)$.

\section{TEMPORAL ONSET DISCREPANCY BETWEEN MOVEMENT AND FEEDBACK}

There was a main effect of feedback condition on onset discrepancy $[F(1,20)=212.45, p<0.001]$. This result, however, should be interpreted as manipulation check only, as executed movement and visual feedback were identical on real feedback trials (i.e., temporal discrepancy $=0$ ), whereas there was an average discrepancy between movement and the false feedback of $351 \mathrm{~ms}(\mathrm{SD}=81 \mathrm{~ms})$. Taking into account how onset discrepancies influenced the experience of false feedback, smaller temporal discrepancies between executed movements and false feedback were associated with incorrect attribution of false feedback [FS $<$ FO, $t(10)=-4.03, p=0.002$; Table 1]. There were no other significant differences between feedback-byrating conditions. Temporal onset discrepancy correlated significantly with accuracy $\left(r_{s}=0.770, p<0.001\right)$.

\section{PHYSIOLOGICAL INDICES}

Skin conductance response was similarly modulated by real ( $M=13.8, \mathrm{SD}=7 \%$ maximum amplitude $)$ or false feedback ( $M=13.6, \mathrm{SD}=7 \%$ maximum amplitude). Thus, no significant effect of feedback on SCRs was found $[F(1,16)=0.17, p=0.899$, n.s.]. Even when taking into account how feedback was experienced, no significant SCR differences between conditions were found (also evident in Table 1). This is supported by Figure 3, which shows that condition-specific SCRs overlap considerably and are not consistently modulated in the single participants. SCR did not correlate significantly with overall accuracy $\left(r_{s}=-0.130\right.$, $p=0.606$, n.s.).

There also was no significant main effect of feedback on changes in $\operatorname{HR}[F(1,16)=1.01, p=0.331$, n.s.]. However, Standard deviations of HR change were high on both real $(M=0.31, \mathrm{SD}=0.88 \mathrm{bpm})$ and false feedback $(M=-0.21, \mathrm{SD}=1.1 \mathrm{bpm})$. Thus, we had a closer look at agency ratings and individual data. False feedback rated as other was, by trend, associated with no change or slowing of HR compared to the other conditions (Table 1), especially compared to correctly attributed real feedback $[\mathrm{FO}<\mathrm{RS}, t(8)=2.661, p=0.029$, n.s.; Figure 5; also visible in six out nine participants in Figure 4]. Changes in HR did not correlate significantly with overall accuracy $(r=0.058, p=0.819$, n.s. $)$. Incidentally, when testing single time bins, at no time point was HR change correlated with feedback or accuracy (all $p>0.376)$.

\section{DISCUSSION}

We sought to identify behavioral and physiological variables that may index the sense of agency, particularly the pre-reflective feeling of agency, while focusing on skin conductance and HR activity. Manipulating the visual feedback to an executed movement, we investigated how well each behavioral and physiological variable differed between real and false feedback conditions (i.e., between factual agency and non-agency), and how they related to the accuracy of agency judgments.

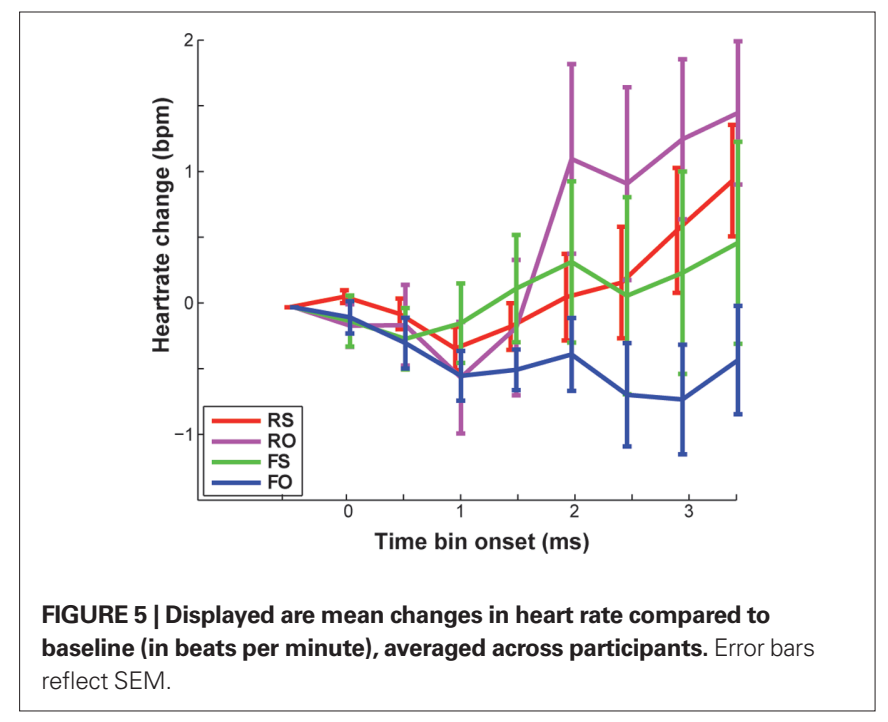

\section{ACCURACY OF AGENCY RATINGS}

Participants successfully reported real feedback, recognizing almost all real feedback trials as self-generated. Their accuracy on false feedback trials, however, differed remarkably from this near-perfect performance: false feedback was incorrectly judged as self-generated on more than half of all false feedback trials. Such vicarious agency has been previously reported (Fourneret and Jeannerod, 1998; Wegner and Wheatley, 1999; David et al., 2007, 2008; Schimansky et al., 2010), suggesting a natural bias toward the experience of self-agency, on the one hand, or insufficiency of verbal reports, on the other. That is, verbal reports may represent subjective and inaccurate measures because they can be subject to errors through misidentification (Shoemaker, 1968). Furthermore, agency reports may only insufficiently assess the pre-reflective, sensorimotor feeling of agency (Tsakiris et al., 2005; Synofzik et al., 2008a,b), as conscious access to this agency level may be limited. Thus, which other variables differ between real and false feedback?

\section{MEASURES OF ACTUAL AGENCY INDEPENDENT OF EXPERIENCE}

Only onset discrepancy between movement and feedback differed significantly on real and false feedback trials. This was intended by our experimental manipulation, similar to previous work that manipulated visual feedback to participants' movements by introducing fixed temporal delays (e.g., 60-270 ms, MacDonald and Paus, 2003; 50-500 ms, Franck et al., 2001). No other measure we had assessed differed between real and false feedback conditions (irrespective of how they were rated). Especially physiological activity, which we had focused on, was not significantly modulated by factual agency (i.e., real vs. false feedback). Skin conductance was almost identical across conditions, even when examined on the singlesubject level. HR - at least, descriptively - slowed on false feedback compared to real feedback (especially when correctly attributed: FO $<$ RS), but this was not significant after multiple-comparison correction. These results indicate weak somatic associations with actual agency. Furthermore, they may be in contrast with evidence showing that self-agency, or real feedback to participants' movements, elicits activation in the anterior insula, which has also been implicated with afferents from somatic or visceral sensations of 
the body and interoceptive awareness but also with motor intention (Roper et al., 1993; Farrer and Frith, 2002; Farrer et al., 2003; Nahab et al., 2011). Roper et al. (1993), for example, showed that lesions to the insula triggered visceral hallucinations as well as unintentional automated movements. Our findings may indicate that, first, physiological activity of the visceral nervous system does not necessarily correspond to neurophysiological activity of the CNS, second, our task was not "somatic enough" (i.e., we investigated effects on disembodied or external events in the visual field) or that, third, the sense of agency does not rely on somatic events (in contrast to, for example, the sense of body ownership - the sense my body is moving, voluntarily or involuntarily; Gallagher, 2000). Yet, Engbert et al. (2007) showed that the sense of agency is not limited to somatic events (i.e., events applied to the participant's body). Other strands of evidence showed that, for example, body-relatd activity such as in the insula is specifically crucial for self-awareness (Karnath et al., 2005) or that somatic markers may inform an active decision-making process (Damasio, 1996). Thus, maybe physiological activity was specifically related to agency judgments?

\section{RELATIONSHIP TO ACCURACY OF AGENCY JUDGMENTS}

Only onset discrepancy correlated significantly with accuracy of agency judgments. The temporal difference between movement and false feedback onset was greater for correct as opposed to incorrect agency judgments (condition FO vs. FS). Thus, onset discrepancies seem to inform agency decisions, which is line with one of the dominant explanatory accounts of action awareness: the forward model of motor control, which predicts sensory consequences of an action via an efference copy, that is, a copy of the motor command (von Holst and Mittelstaedt, 1950). Violations of the predicted or intended state by comparison with the actually observed sensory feedback, then, may lead to the attribution of another agent as the cause of an action (Figure 1), especially the stronger and more salient incongruence (Wolpert et al., 1995). Previous studies (Franck et al., 2001; Leube et al., 2003; MacDonald and Paus, 2003), which used fixed feedback delays, demonstrated that longer delays are associated with increased detection of false feedback. For example, when explicitly instructing participants to attend to onset discrepancies, it has been shown that delays as short as $150 \mathrm{~ms}$ can be detected so that the observed visual event is no longer attributed to the self (Franck et al., 2001). In contrast, Farrer et al. (2008) argued that temporal properties of movements play a secondary role in the sense of agency. We only found partial evidence for Farrer et al. (2008): while here bigger temporal delays did lead to the experience of another agent, onset times of joystick movements alone were not informative for agency. For example, participants may have voluntarily delayed their own movement to test for false feedback. The analyses of onset times only partially supported this idea, as participants indeed initiated their movements later on correctly identified real feedback trials. Nonetheless, onsets did not correlate significantly with overall accuracy. For example, they were not different for correctly

\section{REFERENCES}

Balslev, D., Cole, J., and Miall, R. C. (2007). Proprioception contributes to the sense of agency during visual observation of hand movements: evidence from temporal judgments of action. J. Cogn. Neurosci. 19, 1535-1541.

Bauer, R. M. (1984). Autonomic recognition of names and faces in

and incorrectly evaluated false feedback. It would be interesting to analyze whether participants show other changes in the speed profile of their movements (e.g., whether they slowed down when they noticed a feedback disturbance). Unfortunately, we could not determine this exact moment as ratings were only given after a trial.

Physiological activity also did not correlate with accuracy of agency judgments. Both measures, SCR and HR, were either not at all or not consistently modulated by correctly or incorrectly experienced agency. Moreover, they were not immune to error (e.g., HR did not change differently on correct vs. incorrect self-attributions of feedback). In that sense, decisions about agency seem different from other real-life decisions typically associated with visceralsomatic activity, which may imply more arousal or emotional connotations (Bechara et al., 1996; Damasio, 1996; Crone et al., 2004; Weike et al., 2007).

\section{CONCLUSION}

Here we sought to identify non-verbal measures of the sense of agency. Specifically, we aimed to assess the role of the visceral nervous system, as measured by skin conductance and HR activity, for the feeling of agency. Skin conductance and HR were not differently or consistently modulated between actual agency and non-agency situations. They informed neither the feeling nor judgments of agency. Only discrepancies between movement onset and the onset of false visual feedback were related to the experience of agency. Although our sample size can be considered small and physiological activity is often characterized by high inter-individual variability, we conclude that physiological activity does not represent an empirical indicator for the feeling of agency. Our results indicate only weak visceral-somatic associations with the sense of agency. Thus, despite being a phenomenon often considered closely related to states of the body, the sense of agency may not have a strong somatic basis, in contrast to the sense of body ownership, emotional, or other embodied processes. Instead, it rather seems to depend on the monitoring of movements and their predicted sensory consequences (e.g., here: onset of visual feedback). This is supported by various neuroimaging studies of agency, repeatedly reporting activation in mismatch-sensitive regions such as the parietal lobule (Blakemore and Sirigu, 2003), as well as by investigations of abnormalities in action awareness in patient populations such as schizophrenia (Synofzik et al., 2010). In the context of agency, motor-related measures (e.g., movement durations, movement adjustments, eye movements, etc.) might yield a more promising avenue for future research compared to physiological indices, which may, however, have the potential to tap into the sense of body ownership.

\section{ACKNOWLEDGMENTS}

We acknowledge financial support through the EU projects "MindBridge" (NEST-2006-043457) and "eSMCs" (ICT-270212) awarded to Andreas K. Engel. M. X Cohen is acknowledged for support concerning task programming and I. A. Peiker for statistical advice.

prosopagnosia: a neuropsychological application of the Guilty Knowledge Test. Neuropsychologia 22, 457-469.

Bechara, A., Tranel, D., Damasio, H., and Damasio,A.R.(1996). Failure to respond autonomically to anticipated future outcomes following damage to prefrontal cortex. Cereb. Cortex 6, 215-225.

Blakemore, S. J., and Sirigu, A. (2003). Action prediction in the cerebellum 
and in the parietal lobe. Exp. Brain Res. 153, 239-245.

Bulot, V., Thomas, P., and DelevoyeTurrell,Y. (2007). A pre-reflective indicator of an impaired sense of agency in patients with Schizophrenia. Exp. Brain Res. 183, 115-126.

Castiello, U., Paulignan, Y., and Jeannerod, M. (1991). Temporal dissociation of motor responses and subjective awareness : a study in normal subjects. Brain 114, 2639-55.

Claus, D., and Schondorf, R. (1999). Sympathetic skin response. The International Federation of Clinical Neurophysiology. Electroencephalogr. Clin. Neurophysiol. (Suppl) 52, 277-282.

Crone, E. A., Somsen, R. J., Van Beek, B., and Van Der Molen,M.W.(2004). Heart rate and skin conductance analysis of antecendents and consequences of decision making. Psychophysiology 41, 531-540.

Codispoti, M., Bradley, M. M., and Lang, P. J. (2001). Affective reactions to briefly presented pictures. Psychophysiology 38, 474-478.

Damasio, A. R. (1996). The somatic marker hypothesis and the possible functions of the prefrontal cortex. Philos. Trans. R. Soc. Lond. B Biol. Sci. 351, 1413-1420.

Daprati,E., Franck, N., Georgieff, N.,Proust, J., Pacherie, E., Dalery, J., and Jeannerod, M. (1997). Looking for the agent: an investigation into consciousness of action and self-consciousness in schizophrenic patients. Cognition 65, 71-86.

David, N., Cohen, M. X., Newen, A., Bewernick, B. H., Shah, N. J., Fink, G. R., and Vogeley, K. (2007). The extrastriate cortex distinguishes between the consequences of one's own and others' behavior. Neuroimage 36, 1004-1014.

David, N., Gawronski, A., Santos, N. S., Huff, W., Lehnhardt, F. G., Newen, A., and Vogeley, K. (2008). Dissociation between key processes of social cognition in autism: impaired mentalizing but intact sense of agency. J. Autism Dev. Disord. 38, 593-605.

David, N., Jansen, M., Cohen, M. X., Osswald, K., Molnar-Szakacs, I., Newen, A., Vogeley, K., and Paus, T. (2009). Disturbances of self-other distinction after stimulation of the extrastriate body area in the human brain. Soc. Neurosci. 4, 40-48.

Engbert, K., Wohlschläger, A., Thomas, R., and Haggard, P. (2007). Agency, subjective time, and other minds. J. Exp. Psychol. Hum. Percept. Perform. 33, 1261-1268.

Esslen, M., Metzler, S., Pascual-Marqui, R., and Jancke, L. (2008). Pre-reflective and reflective self-reference: a spatiotemporal EEG analysis. Neuroimage 42, 437-449.

Farrer, C., Bouchereau, M., Jeannerod, M., and Franck, N. (2008). Effect of distorted visual feedback on the sense of agency. Behav. Neurol. 19, 53-57.

Farrer, C., Franck, N., Georgieff, N., Frith, C. D., Decety, J., and Jeannerod, M. (2003). Modulating the experience of agency: a positron emission tomography study. Neuroimage 18, 324-333.

Farrer, C., and Frith, C. D. (2002). Experiencing oneself vs. another person as being the cause of an action: the neural correlates of the experience of agency. Neuroimage 15, 596-603.

Fourneret, P., Franck, N., Slachevsky, A., and Jeannerod, M. (2001). Self-monitoring in schizophrenia revisited. Neuroreport 12, 1203-1208.

Fourneret, P., and Jeannerod, M. (1998) Limited conscious monitoring of motor performance in normal subjects. Neuropsychologia 36, 1133-1140.

Fowles, D. C., Christie, M. J., Edelberg, R., Grings, W. W., Lykken, D. T., and Venables, P. H. (1981). Committee report. Publication recommendations for electrodermal measurements. Psychophysiology 18, 232-239.

Franck, N., Farrer, C., Georgieff, N., MarieCardine, M., Dalery, J., d'Amato, T., and Jeannerod, M. (2001). Defective recognition of one's own actions in patients with schizophrenia. Am. J. Psychiatry 158, 454-459.

Gallagher, S. (2000). Philosophical conceptions of the self: implications for cognitive science. Trends Cogn. Sci. (Regul. Ed.) 4, 14-21.

Georgieff, N., and Jeannerod, M. (1998). Beyond consciousness of external reality: a "who" system for consciousness of action and self-consciousness. Conscious. Cogn. 7, 465-477.

Hamm, A. O., and Vaitl, D. (1996). Affective learning: awareness and aversion. Psychophysiology 33, 698-710.

Karnath, H. O., Baier, B., and Nagele, T. (2005). Awareness of the functioning of one's own limbs mediated by the insular cortex? J. Neurosci. 25, 7134-7138.

Kettunen, J., and Keltikangas-Jarvinen, L. (2001). Intraindividual analysis of instantaneous heart rate variability. Psychophysiology 38, 659-668.

Knoblich, G., and Kircher, T. T. (2004). Deceiving oneself about being in control: conscious detection of changes in visuomotor coupling. J. Exp. Psychol. Hum. Percept. Perform. 30, 657-666.

Legrand, D. (2007). Subjectivity and the body: introducing basic forms of self-consciousness. Conscious. Cogn. 16, 577-582.

Leube, D. T., Knoblich, G., Erb, M., Grodd, W., Bartels, M., and Kircher, T. T. (2003). The neural correlates of perceiving one's own movements. Neuroimage 20, 2084-2090.

Lykken, D. T., and Venables, P. H. (1971). Direct measurement of skin conduct- ance: a proposal for standardization. Psychophysiology 8, 656-672.

MacDonald, P.A., and Paus, T. (2003). The role of parietal cortex in awareness of self-generated movements: a transcranial magnetic stimulation study. Cereb. Cortex 13, 962-967.

Malmivuo, J., and Plonsey, R. (1995). The Electrodermal Response. Bioelectromagnetism: Principles and Applications of Bioelectric and Biomagnetic Fields. New York: Oxford University Press.

Malmstrom, E. J., Opton, E. Jr., and Lazarus, R. S. (1965). Heart rate measurement and the correlation of indices of arousal. Psychosom. Med. 27, 546-556.

Metzinger, T. (2003). Being No One: The Self-Model Theory of Subjectivity. Cambridge, MA: The MIT Press.

Nahab, F. B., Kundu, P., Gallea, C., Kakareka J., Pursley, R., Pohida, T., Miletta, N. Friedman, J., and Hallett, M. (2011). The neural processes underlying selfagency. Cereb. Cortex. 21, 48-55.

Nielsen, T. (1963). Volition: a new experimental approach. Scand. J. Psychol. 4 225-230.

Plesa Skwerer, D., Borum, L., Verbalis, A. Schofield, C., Crawford, N., Ciciolla L., and Tager-Flusberg, H. (2009). Autonomic responses to dynamic displays of facial expressions in adolescents and adults with Williams syndrome. Soc. Cogn. Affect. Neurosci. 4, 93-100.

Ritz, T., Alatupa, S., Thons, M., and Dahme, B. (2002). Effects of affective picture viewing and imagery on respiratory resistance in nonasthmatic individuals. Psychophysiology 39, 86-94.

Roper, S. N., Levesque, M. F., Sutherling, W.W., and Engel, J. Jr. (1993). Surgical treatment of partial epilepsy arising from the insular cortex. Report of two cases. J. Neurosurg. 79, 266-269.

Ruiz-Padial, E., Mata, J. L., Rodriguez, S. Fernandez, M.C., and Vila, J. (2005). Non-conscious modulation of cardiac defense by masked phobic pictures. Int. J. Psychophysiol. 56, 271-281.

Schimansky, J., Roessler, W., David, N., and Haker, H. (2010). Sense of agency and mentalizing: dissociation of subdomains of social cognition in subjects with schizophrenia. Psychiatry Res 178 39-45.

Shergill, S. S., Samson, G., Bays, P. M. Frith, C. D., and Wolpert, D. M. (2005) Evidence for sensory prediction deficits in schizophrenia. Am. J. Psychiatry 162, 2384-2386.

Shoemaker, S. (1968). Self-reference and self-awareness. J. Philos. 65, 555-567.

Slachevsky, A., Pillon, B., Fourneret, P. Pradat-Diehl, P., Jeannerod, M., and Dubois, B. (2001). Preserved adjustment but impaired awareness in a sensory-motor conflict following prefrontal lesions. J. Cogn. Neurosci. 13, 332-340.

Synofzik, M., Lindner, A., and Thier, P. (2008). The cerebellum updates predictions about the visual consequences of one's behavior. Curr. Biol. 18, 814-818.

Synofzik, M., Thier, P., Leube, D. T., Schlotterbeck, P., and Lindner, A. (2010). Misattributions of agency in schizophrenia are based on imprecise predictions about the sensory consequences of one's actions. Brain $133(\mathrm{Pt}$ 1), 262-271.

Synofzik, M., Vosgerau, G., and Newen, A. (2008a). Beyond the comparator model: a multifactorial two-step account of agency. Conscious. Cogn. 17, 219-239.

Synofzik, M., Vosgerau, G., and Newen, A. (2008b). I move, therefore I am: a new theoretical framework to investigate agency and ownership. Conscious. Cogn. 17, 411-424.

Tsakiris, M., Haggard, P., Franck, N., Mainy, N., and Sirigu, A. (2005). A specific role for efferent information in self-recognition. Cognition 96, 215-231.

von Holst, E., and Mittelstaedt, H. (1950). Das Reafferenzprinzip. Naturwissenschaften 37, 464-476.

Wegner, D. M., and Wheatley, T. (1999). Apparent mental causation. Sources of the experience of will. Am. Psychol. 54, 480-492.

Weike, A. I., Schupp, H. T., and Hamm, A. O. (2007). Fear acquisition requires awareness in trace but not delay conditioning. Psychophysiology 44, 170-180.

Wolpert, D. M., Ghahramani, Z., and Jordan, M. I. (1995). An internal model for sensorimotor integration. Science 269, 1880-1882.

Conflict of Interest Statement: The authors declare that the research was conducted in the absence of any commercial or financial relationships that could be construed as a potential conflict of interest.

Received: 27 December 2010; accepted: 19 June 2011; published online: 06 July 2011. Citation: David N, Stenzel A, Schneider TR and Engel AK (2011) The feeling of agency: empirical indicators for a pre-reflective level of action awareness. Front. Psychology. 2:149. doi: 10.3389/ fpsyg.2011.00149

This article was submitted to Frontiers in Consciousness Research, a specialty of Frontiers in Psychology.

Copyright (C) 2011 David, Stenzel, Schneider and Engel. This is an open-access article subject to a non-exclusive license between the authors and Frontiers Media SA, which permits use, distribution and reproduction in other forums, provided the original authors and source are credited and other Frontiers conditions are complied with. 